\title{
ESTRATEGIA DIDÁCTICA PARA LA FORMACIÓN DEL VALOR RESPONSABILIDAD
}

\section{DIDACTIC STRATEGY FOR THE FORMATION OF THE RESPONSIBILITY VALUE}

\section{José Jesús Matos Ceballos \\ jmatos@pampano.unacar.mx}

Currículo: doctor en Ciencias Pedagógicas. Docente de la Universidad Autónoma del Carmen, México. Sus líneas de investigación abordan la cultura física y la orientación educativa en los estilos de vida.

\section{Juan Francisco Tejera Concepción \\ jtejera@ucf.edu.cu}

Currículo: doctor en Ciencias Pedagógicas. Docente de la Universidad de Cienfuegos "Carlos Rafael Rodríguez", Cuba. Sus líneas de investigación abordan la salud física y mental en niños y jóvenes, salud física en la comunidad, estilos y modos de vida.

\section{Carlos Emilio Terry Rodríguez \\ cterry@ucf.edu.cu}

Currículo: doctor en Ciencia de la Cultura Física y el Deporte. Docente de la Universidad de Cienfuegos "Carlos Rafael Rodríguez", Cuba. Sus líneas de investigación abordan el deporte en la comunidad, actividad física y deporte saludable.

Recibido: 29 de agosto de 2017. Aceptado para su publicación: 1 de febrero de 2018. Recuperado de: https://sinectica.iteso.mx/index.php/SINECTICA/article/view/786 DOI: 10.31391/S2007-7033(2018)0050-013

\section{Resumen}

El artículo tiene como objetivo presentar la propuesta de una estrategia didáctica para la formación del valor responsabilidad en los estudiantes de la licenciatura en Educación Física y Deporte en la Universidad Autónoma del Carmen, México. En la investigación que da origen a este trabajo se aplicaron diversas técnicas y métodos en el diagnóstico inicial del estado de la formación del valor responsabilidad, la elaboración del marco teórico, el desarrollo de la estrategia didáctica y su aplicación en la citada profesión. Los resultados señalan la viabilidad de aplicar los elementos teóricos y prácticos que constituyen las aportaciones del estudio, con sus correspondientes adecuaciones, a otros contextos universitarios, así como en el proceso formativo de posgrado.

Palabras clave: estrategia didáctica, licenciatura en educación física y deporte, valor responsabilidad.

\footnotetext{
Abstract

The present investigation has aimed propose a didactic strategy for the formation of the responsibility value in the students of the Degree in Physical Education and Sport. The investigation transited, by means of diverse techniques and methods of investigation, for the initial diagnosis of the status of the formation of the responsibility value, the elaboration of the theoretical framework, at the autonomous University of Carmen, Mexico. The theoretical and practical
} 
elements that constitute contributions of work, with its corresponding adjustments, they are also applicable in other university contexts, as well as in the training process in graduate school.

Keywords: didactic strategy, bachelor's degree in physical education and sport, value responsibility.

\section{INTRODUCCIÓN}

a Organización de las Naciones Unidas para la Educación, la Ciencia y la Cultura (Unesco, 2015) recomienda que los programas de formación del profesional en educación física hagan hincapié en la formación de valores asociados a la práctica de la educación física y el deporte, como el respeto, el juego limpio y la tolerancia. Entre los investigadores que han abordado el tema de los valores en el ámbito educativo, están Fabelo (2004); Bauzá y Marañón (2012); Benítez, Díaz, Pozo, Vargas y Núñez (2012); Navarro (2012); Castro, Chacón y Mendoza (2012); y Gómez, Quintana, Medina, Hernández y Beristaín (2013). Estos autores coinciden en que en el ámbito pedagógico los valores se adquieren en la dimensión social de la práctica educativa a través de la interacción; ello, en función de la organización de los contenidos y las prácticas metodológicas, los cuales, posteriormente, son integrados y socializados como proceso.

En el ámbito internacional, encontramos los trabajos de Domínguez (1996), Zaldívar (2004), Collado (2005), Ojalvo (2006), González, Blández, López y Sierra (2007), y Sivira (2011), quienes centran sus análisis en la formación del valor responsabilidad en el área de educación física y deporte, y demuestran la tendencia cada vez más marcada de encauzarse como proceso de estructuración de la personalidad. En México, Torres y Guerrero (2012) coordinaron un estudio sobre la formación de valores en educación física, conceptualizados en la ética del maestro, desempeño del docente, tolerancia, respeto, compromiso, honestidad, disciplina y equidad en las enseñanzas primaria, media y media superior. No identificamos investigaciones cuyo objetivo fuera la integración de contenidos y actividades de la práctica profesional para la formación del valor responsabilidad en el área de la educación física y el deporte en la enseñanza superior.

\section{Situación de la problemática}

El análisis de la literatura consultada revela la poca importancia que algunos docentes otorgan a los modos de actuación de los estudiantes en las actividades docentes y su escasa corrección en el contexto universitario. Esto concuerda con el estudio empírico que realizamos, que arroja la pobre preparación que los alumnos de la licenciatura en Educación Física y Deporte de la Universidad Autónoma del Carmen tuvieron en cuanto a la formación del valor responsabilidad.

Las insuficiencias en los modos de actuación de estos alumnos asociadas al valor responsabilidad se traducen en impuntualidades en las actividades académicas y en la práctica profesional; uso incorrecto del uniforme escolar; dificultades con el porte y aspecto personal; inadecuado desarrollo de la expresión oral; falta de organización en la recogida de los implementos deportivos e insuficiencias en las relaciones interpersonales y empáticas con sus compañeros y otros semejantes.

Esta situación nos condujo al planteamiento de la siguiente pregunta: ¿cómo contribuir a la formación del valor responsabilidad en los estudiantes de la licenciatura en Educación Física y Deporte? 


\section{DiSEÑO DE INVESTIGACIÓN}

Como respuesta a la pregunta de investigación, nuestro objetivo fue proponer una estrategia didáctica para la formación del valor responsabilidad en los estudiantes de la citada licenciatura. Las tareas consistieron en la determinación de los presupuestos teóricos para la formación del valor responsabilidad; el diagnóstico del estado actual sobre la formación del valor responsabilidad en estos estudiantes; la elaboración de una estrategia didáctica para la formación del valor responsabilidad; la valoración de esta estrategia mediante el criterio de expertos y su aplicación en la práctica.

\section{Estructura metodológica}

El estudio es de tipo cualitativo, con la utilización de algunos métodos y técnicas de tipo cuantitativo, según fue necesario en cada momento del proceso. Los métodos y técnicas empleados fueron:

Nivel teórico

- Histórico-lógico (para el análisis acerca de la evolución del objeto de estudio en el contexto nacional e internacional y para determinar su estado actual).

- Inductivo-deductivo (de acuerdo con los nexos entre estas dos formas de inferencia lógica para formular las conclusiones).

-Analítico-sintético (para descomponer las complejidades de los procesos cognitivos, determinar las relaciones entre ellos, recopilar elementos importantes, estudiar la información documental y la situación problemática).

- Sistémico-estructural (para garantizar la estructura de la estrategia didáctica).

Nivel empírico

-Análisis de documentos de la licenciatura.

- Entrevista a egresados de la licenciatura en Educación Física y Deporte.

- Cuestionario a los estudiantes.

- Criterio de expertos.

- Técnica de Ladov para conocer el grado de satisfacción de los estudiantes en la aplicación de la estrategia didáctica.

- Observación participante en diferentes actividades de la práctica profesional con la finalidad de evaluar y obtener información acerca del valor responsabilidad.

- Técnica estadística para el análisis y la interpretación de los datos obtenidos como resultado de los métodos y las técnicas aplicadas.

La contribución a la teoría consistió en el procedimiento metodológico para realizar la integración de los contenidos de las asignaturas Educación física, Entrenamiento deportivo y actividades de la práctica profesional. Por su parte, el aporte práctico se reflejó en la elaboración de la citada estrategia didáctica sustentada en la integración de contenidos de las asignaturas y actividades de la práctica profesional. 
La propuesta representa una novedad científica por ser una respuesta educativa a la calidad de la licenciatura en Educación Física y Deporte, en la formación integral y el carácter didáctico que se le confiere para la formación de valor responsabilidad en los estudiantes, lo que les permite un adecuado desempeño profesional mediante el cumplimiento de sus funciones en el contexto universitario.

\section{MARCO TEÓRICO}

Este apartado contiene los fundamentos teóricos que permitieron trabajar el objeto de la investigación y nos llevaron a definir los elementos que abordan la formación del valor responsabilidad en los estudiantes de la mencionada licenciatura.

\section{Fundamentación teórica sobre los valores}

De acuerdo con el paradigma de Nietzsche, citado por Collado (2005), el hombre concede valores a las cosas para sostenerse a sí mismo; fue él quien primero asignó un sentido de las cosas, un sentido de lo humano. De ello, podemos deducir que la tarea de una educación moral es poner y crear valores que llenen el sentido humano a las cosas, las acciones y realizaciones que guardan relación con el hombre y que este mismo efectúa.

Consideramos que los modos de actuación y las conductas en los diferentes contextos de la vida son condicionados por lo personológico como ente social. Moleiro (2001) expresa que las cosas no son valiosas por sí mismas, sino que tienen el valor que nosotros les damos y por eso cada persona tiene su propia escala de valores. Asimismo, específica que no todos nos comportamos igual ante las vivencias y los problemas de la vida; según los valores a los que les damos prioridad, le damos sentido a lo que hacemos. Los valores son los ejes fundamentales por los que se orienta la vida humana y constituyen, a su vez, la clave del comportamiento de las personas (Izquierdo, 2003).

Para Guevara (2007), los valores son construcciones humanas, adquiridas a través del proceso socializador del grupo al que se pertenece (familiar, educativo, religioso, político, laboral, entre otros), pues el viviente humano en su variada vida de relación los aprende de personas que le son significativas.

Para Sanders (2005), los valores son conceptos abstractos que conllevan complejidad en su definición, medición e incluso explicación. Los valores, sean generales o particulares, están fuertemente impregnados con la cultura en la que se dan; si bien hay valores denominados universales, hay otros que dependen de su situación geográfica. Los valores cambian con las generaciones y son adyacentes a las leyes basadas en valores; estos son el elemento que cohesiona y norma a las sociedades, les da forma y límite. Los valores están siempre presentes, si bien invisibles, determinan en forma cotidiana, y a largo plazo, el rumbo de las sociedades en particular y de la humanidad en general.

García, (2003), Fabelo (2004) y Díaz (2011) consideran que los valores permiten resaltar aspectos significativos con relación a la comprensión de su esencia, entre los que se encuentran los componentes que intervienen en su proceso de formación:

-Unidad dialéctica entre los aspectos objetivos y subjetivos; lo individual y lo social.

-Son construidos por los individuos dentro de sus correspondientes grupos, de acuerdo con sus necesidades, intereses y vivencias. 
- Ejercen función reguladora de la actividad humana.

- Su aprendizaje es relativamente estable en el tiempo y permiten comparar normas de actuación.

- Se manifiesta como un fenómeno sociohistórico y se sustenta en el aspecto interactivo del sujeto y el objeto.

- Es todo aquello que es deseable o interesa a un sujeto en función de su carácter o por su concepción como satisfactor de una necesidad e influye en su conducta de elección.

La necesidad del estudio de los valores ha existido siempre y responde a los intereses de clases y a las condiciones concretas. Por ello, se hace necesario conocer el estudio teórico de los valores para aunar las influencias educativas que permitan mantener las conquistas sociales logradas.

\section{Definiciones sobre el valor responsabilidad}

El valor responsabilidad es la cualidad de la acción que hace posible que a las personas se les pueda demandar que actúen moralmente y cómo los hombres y las mujeres son responsables de sus actos; se les puede pedir cuentas de por qué lo hacen y también de los efectos que de esas acciones se derivan para las otras personas o para la naturaleza (Escámez, 2001). La responsabilidad, como manifestación de la personalidad, es un fenómeno complejo y se expresa en tan variadas situaciones que puede llegar a determinar la vida exitosa de un individuo (León, 2011). La responsabilidad implica tomar las riendas de la propia vida y esta se concreta en acciones específicas de compromiso; por tanto, no existe la responsabilidad en abstracto, lo que existe son acciones comprometidas en la vida cotidiana de cada persona (Reyes, 2014).

Hay que tener presente que un estudiante universitario es responsable no porque reconozca la importancia del valor responsabilidad por las circunstancia que lo obligan a ser responsable, sino porque sienta la necesidad de actuar de manera responsable (Fabelo, 2004). La responsabilidad es un motivo de la actuación del sujeto y se convierte en un verdadero regulador de su conducta.

La responsabilidad es desarrollar una constelación de valores: la autoestima, sinceridad, perseverancia, iniciativa, fortaleza, generosidad, respeto, confianza, lealtad (Collado, 2005). Esto nos conduce a reconocer las dos vertientes implicadas: la individual y la colectiva; no solo reconocemos y aceptamos las consecuencias de nuestros propios actos, sino también influimos en las decisiones de un conjunto de personas y somos capaces de responder por las que toma el grupo al que pertenecemos.

La educación de la responsabilidad como valor profesional constituye hoy una necesidad insoslayable si se tiene en cuenta que la sociedad actual exige un profesional que se desempeñe con autonomía, competencia y flexibilidad en escenarios laborales complejos y diversos (González, Blández, López y Sierra, 2007).

Asumimos, en el mismo sentido de Paz, Comas y Fernández (2011), que un estudiante será responsable cuando la labor pedagógica se ejecuta sistemática y regularmente, no "de vez en cuando"; que el contenido de cada actividad se adecue al grupo al que va dirigida; que los métodos que se utilicen estimulen la autoeducación y la reflexión de los alumnos; y que su aplicación se conciba en un marco de calor humano alejada de toda improvisación. 
Otros investigadores también han realizado diversos estudios sobre este tema, como Domínguez (1996), quien llevó a cabo una experiencia pedagógica basada en técnicas participativas y métodos en los que se articulaban los grupos de trabajo científico estudiantil sobre la base de la concepción histórica-cultural de la actividad. Por su parte, Ojalvo (2002) elaboró un programa de formación docente para el diseño de estrategias educativas curriculares dirigidas a potenciar el fomento de la responsabilidad en los estudiantes universitarios.

Zaldívar (2004) construyó un modelo de organización del trabajo metodológico para contribuir a educar el valor responsabilidad en estudiantes universitarios de la cultura física mediante las potencialidades axiológicas de una estrategia educativa, y Collado (2005) aplicó un programa de intervención sociomotriz basado en juegos cooperativos para la formación del valor responsabilidad, respeto y autoestima en estudiantes de secundaria básica. González, Blández, López y Sierra (2007) abordaron el valor responsabilidad en alumnos de licenciatura en Educación Física mediante una estrategia educativa que centró su atención en la práctica laboral.

Los estudios descritos demuestran la tendencia cada vez más acentuada de comprender la formación del valor responsabilidad como un proceso de desarrollo de la personalidad en los estudiantes y, por lo tanto, la necesidad de elaborar diferentes métodos educativos dirigidos a potenciar su desarrollo.

\section{Conceptualizaciones sobre la integración de contenidos}

La integración de contenidos se manifiesta tanto en el nivel macro (en el diseño curricular de la carrera) como en el micro (en la asignatura y la clase); esto coincide con los criterios de Perera (2000), quien plantea que la integración de los contenidos como resultado de la interdisciplinariedad se da en el plano curricular, didáctico y pedagógico. Addine (2006), Licea (2006) y Jiménez (2007) destacan para el proceso de integración un enfoque profesional pedagógico basado en el planteamiento y la solución de problemas desde el modo de actuación profesional, como eje integrador del proceso.

En nuestra investigación adoptamos el concepto brindado por Perera (2000), para quien la integración constituye un basamento teórico esencial, ya que permite orientar la actividad de los profesores y los estudiantes hacia la solución de los problemas profesionales; de este modo, se logra una formación integrada, científica y contextualizada con la época, en correspondencia con las necesidades y exigencias de la sociedad.

\section{Fundamentación didáctica para la formación del valor responsabilidad}

La didáctica es la ciencia que estudia como objeto el proceso de enseñanza- aprendizaje dirigido a resolver la problemática que se le plantea a la escuela: la preparación del hombre para la vida y cuya función es formar al hombre, pero de un modo sistémico y eficiente (Álvarez, 1998). Una de las direcciones de la formación de valores es convertirlo en un proceso accesible y de apoyo a los docentes y en un instrumento activo para cada estudiante, que le permita ser sujeto, objeto y sustantivo a la vez. También constituye una base teórica que sustenta la formación del valor responsabilidad como proceso socializado que abarca desde lo concreto a lo especial.

De lo anterior no escapa el proceso de formación del valor responsabilidad, cuya periodización se garantiza al relacionar los componentes de este proceso con los 
elementos que a él tributan para, así, elaborar una zona de desarrollo potencial a partir de un diagnóstico que haga posible planear, ejecutar y evaluar con certeza el proceso de formación del valor responsabilidad en los estudiantes de la citada licenciatura desde los elementos generales hasta sus particularidades individuales.

El proceso de enseñanza-aprendizaje para la formación del valor responsabilidad requiere un estudio profundo de sus componentes esenciales. Este debe estar sustentado en la integración de los contenidos de las asignaturas y las actividades de la práctica profesional. De acuerdo con Álvarez (1998), en el contenido se revelan tres dimensiones: conocimientos, que reflejan el objeto de estudio; habilidades, que recogen el modo en que se relaciona el hombre con dicho objeto; $y$ valores, que expresan la significación que el hombre le asigna a dichos objetos. Esas tres dimensiones se deben interpretar como tipos de contenidos distintos, cada uno con su propia personalidad; sin embargo, no existen independientes unos de otros, sino que todos se interrelacionan en forma dialéctica por medio de una triada y conforman una unidad que, justamente, es el componente estudiado.

La concepción didáctica de los contenidos se da mediante la integración del sistema de conocimientos y habilidades de las asignaturas de Educación física, Entrenamiento deportivo y actividades de la práctica profesional con el valor responsabilidad como sistema de valores en los estudiantes de la licenciatura en Educación Física y Deporte.

\section{ESTRATEGIA DIDÁCTICA PARA LA FORMACIÓN DEL VALOR RESPONSABILIDAD EN LOS ESTUDIANTES DE LA LICENCIATURA EN EDUCACIÓN FÍSICA Y DEPORTE}

Las estrategias didácticas, según Feo (2010), se definen como los procedimientos (métodos, técnicas, actividades) mediante los cuales el docente y los estudiantes organizan las acciones en forma consciente para construir y lograr metas previstas e imprevistas en el proceso enseñanza-aprendizaje, y se adaptan a las necesidades de los participantes de modo significativo.

Valle (2007) precisa la estrategia en el ámbito de la pedagogía con el objetivo de lograr cierta unidad en su utilización. Para este autor, la estrategia didáctica es el conjunto de acciones secuenciales e interrelacionadas que, partiendo de un estado inicial y considerando los objetivos propuestos, permite dirigir el desarrollo del proceso de enseñanza-aprendizaje en la escuela.

En nuestro procedimiento metodológico para la integración de contenidos se aprecia la orientación grupal e individual en la formación del valor responsabilidad; en este sentido, tomamos como referencia a Pherson (2004).

Procedimiento metodológico para la integración de los contenidos en la estrategia didáctica

De forma coherente, organizada y uniforme, realizamos un trabajo de selección de las asignaturas y los contenidos y su integración para la formación del valor responsabilidad en los estudiantes de la licenciatura en Educación Física y Deporte. Nos basamos en el estudio llevado a cabo por el colectivo de profesores de la Universidad de Pinar de Río, de Cuba, sobre la integración de los contenidos energéticos básicos en las disciplinas de física. Hicimos algunas adecuaciones y ajustes a los contenidos de las asignaturas de Educación física, Entrenamiento deportivo y actividades de la práctica profesional para la formación del valor responsabilidad.

La metodología propuesta abarcó tres etapas fundamentales: en la primera llevamos a cabo la exploración de los contenidos. En ella caracterizamos las asignaturas 
ya mencionadas para conocer la disposición y el dominio del tema. Con este propósito, emprendimos las siguientes acciones:

- Reunión con los profesores de los órganos metodológicos de la licenciatura para determinar las posibilidades que brinda cada asignatura y sus contenidos para la integración.

- Elaboración de instrumentos que permitieran aplicar la técnica de la entrevista no estructurada grupal e individual. Los indicadores fueron:

»Dominio de los objetivos formativos de la licenciatura »Objetivos de la integración

«Selección de los profesores para realizar la integración »Preparación de los profesores para realizar la integración »Ejecución de la integración »Información ante el colectivo de las dos academias sobre el resultado de la integración

»Presentación ante el colectivo de las dos academias sobre el impacto que tuvo el proceso de integración

En la segunda etapa de ejecución, las tareas y acciones diseñadas propiciaron la articulación de los contenidos y las asignaturas, lo cual posibilitó el cumplimiento exitoso del proceso. Para ello, llevamos a cabo las siguientes acciones:

- Seleccionamos las asignaturas en reuniones con las dos academias.

- Elegimos los contenidos mediante la consulta con los profesores.

- Consultamos a los profesores en una discusión grupal para valorar la pertinencia de la integración y el cumplimiento de los objetivos propuestos. Elaboramos un informe conclusivo.

La tercera etapa, de evaluación, consistió en la integración realizada: asignaturas, contenidos y valoración del proceso de integración como un todo. Presentamos a los órganos metodológicos los resultados obtenidos en la integración, es decir, los contenidos integrados por asignaturas. Para ello recurrimos al diálogo, la discusión y la reflexión compartida, que propiciaron la exigencia, apertura, flexibilidad y libertad con visión crítica de los participantes. Fue un proceso continuo que abarcó las etapas anteriores y definió momentos y acciones.

\section{Planteamiento de la estrategia didáctica para la formación del Valor RESPONSABILIDAD}

La estrategia didáctica consta de cuatro etapas fundamentales: diagnóstico, planeación, ejecución y evaluación. El trabajar cada una armónicamente, unas con otras, en el sistema de contenidos de las asignaturas Educación física, Entrenamiento deportivo y las actividades de la práctica profesional, contribuye a formar el valor responsabilidad en el futuro profesional de la licenciatura en Educación Física y Deporte.

\section{Primera etapa: diagnóstico}

En el diagnóstico no solo se tienen en cuenta las vivencias, experiencias y reflexiones de los estudiantes sobre la formación del valor responsabilidad, sino que se 
produce una retroalimentación a partir de las opiniones y los criterios emitidos por ellos mismos en las diferentes acciones que se ejecutan en esta etapa. El objetivo es establecer el nivel de desarrollo del valor responsabilidad en los estudiantes de la licenciatura. Las acciones a desarrollar son:

-Diagnóstico para determinar el nivel de formación que poseen los estudiantes relacionados con el valor responsabilidad.

-Selección de los métodos y las técnicas que serán utilizadas en el diagnóstico.

-Análisis por parte de los profesores que conforman el cuerpo académico de la licenciatura sobre los resultados obtenidos durante la etapa de diagnóstico a los estudiantes.

-Análisis de documentos:

»Permite analizar los contenidos que se seleccionarán para formar parte de la estrategia didáctica correspondientes a las dos academias (Educación física y Entrenamiento deportivo), actividades de la práctica profesional, su influencia y las posibilidades que brindan estos para la integración y su contribución a la formación del valor responsabilidad.

-Aplicación de instrumentos y técnicas:

„Cuestionario: el objetivo es conocer la opinión de los estudiantes sobre la formación del valor responsabilidad.

„Encuesta: su propósito es determinar el nivel de conocimiento que poseen los estudiantes sobre la formación del valor responsabilidad. "Técnica "Solución de problemas": su objetivo es conocer la capacidad de resolver y analizar las diferentes situaciones problemáticas presentadas en las actividades de la práctica profesional.

- Revisión en la planificación de las actividades de la práctica profesional y la integración con el componente académico que contribuya a la formación del valor responsabilidad.

- Conocer el criterio de los profesores sobre la integración de los contenidos y las actividades de la práctica profesional.

\section{Segunda etapa: planeación}

La etapa de planeación se realiza a partir de los resultados obtenidos en el diagnóstico. Se define el sistema de acciones que promuevan la transformación del objeto desde el estado inicial al deseado. El objetivo es planificar las acciones a ejecutar que posibiliten la formación del valor responsabilidad en los estudiantes de la licenciatura en Educación Física y Deporte.

Las acciones a desarrollar son:

- Preparación de los profesores de las dos academias Educación física y Entrenamiento deportivo en la integración de los contenidos para la formación del valor responsabilidad.

- Selección y preparación de los escenarios donde el estudiante desarrollará las actividades académicas y la práctica profesional.

- Organización de los contenidos y las actividades de la práctica profesional para llevar a cabo el proceso de integración. 
- Organización del trabajo metodológico de la licenciatura con sus órganos académicos.

- Operaciones:

»Analizar los resultados del diagnóstico realizado a los estudiantes. »Construir las propuestas de solución a estudiantes según las necesidades educativas.

»Seleccionar los diferentes escenarios educativos.

»Planificar, con talleres, debates, estudio de casos y el instrumento láminas de responsabilidad, actividades en las que se manifieste la responsabilidad.

»Planear actividades metodológicas en las que se aborde la integración de los contenidos para la formación del valor responsabilidad. "Seleccionar los profesores más competentes para que impartan actividades metodológicas en academias relacionadas con el procedimiento metodológico para la integración de los contenidos.

Tercera etapa: ejecución

El objetivo es ejecutar las acciones de la etapa de planeación. Las operaciones son:

- Examinar, en el trabajo de las dos academias, los resultados del diagnóstico aplicado a los estudiantes.

- Organizar talleres, debates, estudio de casos e instrumento láminas de responsabilidad con estudiantes, en los que se exponga el procedimiento metodológico para la integración de los contenidos.

- Visitar clases modelos y actividades de la práctica profesional con el objetivo de observar las manifestaciones conductuales, su corrección y factibilidad en la contribución a la formación del valor responsabilidad, asociado a los modos de actuación.

- Efectuar actividades metodológicas con las academias en las que se aborde el análisis del procedimiento metodológico en la integración de los contenidos para la formación del valor responsabilidad.

- Realizar intercambios con profesores que pertenecen al sistema de tutorías con los demás docentes para conocer sus impresiones sobre los avances que se reconocen en cuanto a las actividades llevadas a cabo sobre la integración de los contenidos para la formación del valor responsabilidad.

\section{Cuarta etapa: evaluación}

En esta etapa se asume la evaluación de la estrategia como proceso y resultado a la vez, por lo que es necesario considerar qué se obtiene y de qué manera como base para el logro de los objetivos propuestos; por tanto, la evaluación es sistemática y aunque no aparece de manera explícita en las etapas, es evidente que cada una de ellas la prevé.

Esta etapa no se limita a un tiempo determinado, sino que es concebida en toda la estrategia y en cada una de sus etapas, desde el diagnóstico hasta la fase final, pues se hace necesario evaluar en todo momento y retroalimentar y corregir el camino. El objetivo es evaluar las acciones planificadas en la estrategia didáctica para la formación del valor responsabilidad en los estudiantes. 
Las acciones a desarrollar son:

- Evaluación de la efectividad de las acciones propuestas en cada etapa de la estrategia didáctica.

-Verificación del cumplimiento de los objetivos en cada etapa de la estrategia didáctica.

- Operaciones:

»Elaborar situaciones en la que demuestren los modos de actuación asociados al valor responsabilidad.

»Observar el desempeño profesional de los estudiantes en la práctica profesional para verificar si en su formación han interiorizado los modos de actuación que demuestren el valor responsabilidad. »Participar en reuniones metodológicas de las dos academias para constatar la vinculación de las formas docentes utilizadas en la integración de los contenidos.

„Evaluar con los profesores las formas que han utilizado en la selección y organización de los contenidos de acuerdo con sus posibilidades para la integración.

„Evaluar con los profesores de los órganos académicos el desarrollo de las actividades metodológicas planificadas en las que se haya abordado la utilización del procedimiento metodológico para la integración de los contenidos y las actividades de la práctica profesional.

\section{RESULTADOS}

\section{Criterio de selección de la muestra}

Para realizar el estudio, seleccionamos una muestra de estudiantes, profesores y egresados del programa educativo ya mencionado. En cuanto a los estudiantes, de una población general actual de 92 estudiantes de la licenciatura en Educación Física y Deporte, tomamos una muestra acorde con la fórmula estadística establecida como máxima de proporciones, que supone $\mathrm{p}=\mathrm{q}=0.5$ con un nivel de error $\mathrm{B}=0.04$, con un valor calculado que arrojó una cifra de 79 estudiantes a seleccionar según muestreo aleatorio simple. Elegimos estudiantes de los ciclos escolares 2014-2016, en correspondencia con el periodo de ejecución de la investigación.

También elegimos 22 profesores de 55, y 29 egresados de un total de 85 . En cuanto a los primeros, de una población de 55, tomamos una muestra de proporciones, con $\mathrm{p}=\mathrm{q}=0.5$ y error de muestreo de 0.012 , calculando $\mathrm{n}=22$ profesores, seleccionados por muestreo aleatorio simple, correspondientes a los ciclos escolares 2014-2016. En cuanto a los egresados, de una población compuesta por 85 egresados de la licenciatura, tomamos una muestra de 29 , correspondientes a diferentes generaciones de estudio, que fue obtenida a través de un muestreo aleatorio simple, con $\mathrm{p}=\mathrm{q}=0.5$ y un error de 0.11 .

\section{Caracterización de los expertos}

La metodología utilizada para la obtención del criterio de expertos responde a lo planteado por Cortés e Iglesias (2004), quienes ponderan el valor de estos como fuente de pronóstico. Seleccionamos 19 expertos pertenecientes a varias instituciones del sector público, privado y social con base en los siguientes criterios: años de experiencia laboral, perfil laboral docente, grado científico, dominio sobre el 
tema de investigación, y demostrar una destacada labor investigativa. Consideramos que los 19 expertos poseían conocimientos y argumentación suficientes para un coeficiente de competencia alto; por ello, fueron aceptados para la investigación. El coeficiente k de los 19 expertos en forma global es cercano a 1.

\section{Valoración de la estrategia didáctica. Resultados y contribución}

En este apartado ponemos de manifiesto la utilización de métodos, técnicas e instrumentos y las valoraciones relacionadas con la estrategia didáctica; además, analizamos los resultados alcanzados durante la aplicación de esta y su contribución para la formación del valor responsabilidad en los estudiantes de la licenciatura en Educación Física y Deporte de la Universidad Autónoma del Carmen, México.

a) Aplicación del método Delphi para valorar la estrategia didáctica

Para la aplicación de este método efectuamos los cálculos correspondientes a la evaluación de los criterios sobre la valoración de la estrategia didáctica para la formación del valor responsabilidad en los estudiantes de la licenciatura. El objetivo fundamental del método Delphi utilizado en la investigación fue que los expertos evaluaran un conjunto de criterios según la escala de Liker. Para ello, contamos con 19 expertos seleccionados y 17 criterios para evaluar la estrategia. Organizamos el trabajo en tres rondas:

- Primera ronda: proporcionamos a los expertos una lista de criterios importantes sobre la estrategia didáctica, los cuales fueron resultado del diagnóstico inicial durante el proceso investigativo. De manera anónima, los expertos se dieron a la tarea de analizar la propuesta presentada con el objetivo de que se pudieran incorporar nuevas ideas o se concordara con las presentadas o, en su defecto, se eliminara alguna en caso de no llegar a conclusiones.

- Segunda ronda: facilitamos a los expertos el resultado con las ideas reconstituidas en la ronda anterior; posteriormente, hicimos un análisis para su aprobación con la firme intención de encontrar una correspondencia de criterios entre los integrantes del grupo de expertos.

- Tercera ronda: elaboramos una nueva versión de la estrategia didáctica, la cual fue entregada a cada uno de los expertos para que la evaluaran de acuerdo con la escala de Likert; después llevamos a cabo el procesamiento estadístico.

A continuación presentamos los resultados porcentuales en cada criterio de la evaluación de los expertos:

- $100 \%$ consideraron la coherencia en la estructura de la estrategia didáctica como muy adecuada.

- $94 \%$ consideraron muy adecuada y $6 \%$, bastante adecuada la utilidad de las orientaciones metodológicas dadas en cada etapa de la estrategia. - $89 \%$ consideraron muy adecuado y $11 \%$, bastante adecuado el impacto con los indicadores seleccionados para mejorar los modos de actuación que contribuyan a la formación del valor responsabilidad. 
- $73 \%$ calificaron como muy adecuado, $21 \%$, bastante adecuado y $6 \%$, adecuado el criterio de que se aprecia la integración en los contenidos de las asignaturas Educación física, Entrenamiento deportivo y actividades de la práctica profesional, seleccionados en cada etapa de la estrategia didáctica. - $89 \%$ consideraron muy adecuada y $11 \%$, bastante adecuada la relación entre las etapas y las acciones planificadas.

- $79 \%$ estimaron muy adecuada y $21 \%$, bastante adecuada la consideración que se tiene de los indicadores que conforman los modos de actuación para la planificación de las actividades en las diferentes maneras de organización docente que contribuyen a la formación del valor responsabilidad.

- $73 \%$ consideraron muy adecuado, $21 \%$, bastante adecuado y $6 \%$, adecuado el impacto en las mejoras de los modos de actuación para contribuir a la formación del valor responsabilidad.

- $83 \%$ estimaron muy adecuado, $11 \%$, bastante adecuado y $6 \%$, adecuado el criterio de que las etapas y acciones diseñadas responden a los propósitos de la estrategia.

- $94 \%$ valoraron como muy adecuada y $6 \%$, bastante adecuada la fundamentación en cada etapa de la estrategia.

- $68 \%$ consideraron muy adecuado, $21 \%$, bastante adecuado y $11 \%$, adecuado el criterio de que la factibilidad y flexibilidad en la estrategia brinda posibilidades de incorporar nuevas propuestas.

- $89 \%$ calificaron como muy adecuada y $11 \%$, bastante adecuada la fundamentación desde las diferentes áreas del conocimiento, la filosofía, la sociología, la psicología y la didáctica.

- $83 \%$ consideraron muy adecuado y $17 \%$, bastante adecuado el criterio sobre la actualidad de los contenidos seleccionados y que se ajustan a lo establecido por la Universidad Autónoma del Carmen, México.

- $94 \%$ estimaron como muy adecuada y $6 \%$, como bastante adecuada la asequibilidad de la estrategia para ser aplicada en otras carreras de la Universidad.

- $77 \%$ consideraron muy adecuado, $17 \%$, bastante adecuado y $6 \%$, adecuado el criterio de que la estrategia es pertinente y valiosa para el desempeño del futuro profesional.

- $83 \%$ estimaron como muy adecuado y $17 \%$, bastante adecuado el criterio de que las acciones diseñadas se relacionan con el objetivo general y los específicos en cada etapa de la estrategia.

- $94 \%$ calificaron como muy adecuado y $6 \%$, bastante adecuado el criterio de que se pueden derivar otras actividades docentes para mejorar la calidad del proceso de enseñanza-aprendizaje.

- $89 \%$ consideraron muy adecuado y $11 \%$, bastante adecuado el criterio sobre la vigencia de la estrategia en relación con el modelo de formación del futuro egresado de la licenciatura en Educación Física y Deporte.

b) Técnica de Ladov

Utilizamos un cuestionario para conocer el grado de satisfacción de los estudiantes en la aplicación de la estrategia; la muestra se integró de 23 estudiantes (de una población de 92) del cuarto al octavo semestre de la licenciatura mencionada y fue de tipo aleatorio simple. 
A continuación presentamos los resultados porcentuales en cada ciclo escolar sobre el grado de satisfacción:

- En el cuarto ciclo, 41\% declararon estar claramente satisfechos; $34 \%$, más satisfechos que insatisfechos; $17 \%$ no tuvieron criterio; $4 \%$ más insatisfechos que satisfechos; y $4 \%$, clara insatisfacción.

- En el quinto ciclo, $60 \%$ dijeron estar claramente satisfechos; $26 \%$ más satisfechos que insatisfechos; y $14 \%$ no tuvieron criterio.

- En el sexto ciclo, $75 \%$ declararon estar claramente satisfechos; $21 \%$, más satisfechos que insatisfechos; y $4 \%$ no tuvieron criterio.

-En el séptimo y octavo ciclo, $100 \%$ dijeron estar claramente satisfechos.

Estos resultados demuestran que, a medida que se fue aplicando y perfeccionando la estrategia didáctica, el grado de satisfacción en los estudiantes fue aumentando.

c) Análisis de los resultados en la observación participante en las actividades de la práctica profesional para la valoración de la estrategia didáctica

Observamos 39 actividades de la práctica profesional correspondientes a los periodos escolares 2014-2016. Las gráficas 1 y 2 dan cuenta de los resultados porcentuales antes y después de aplicar la estrategia en las actividades.

Gráfica 1. Resultados previos a la aplicación de la estrategia didáctica

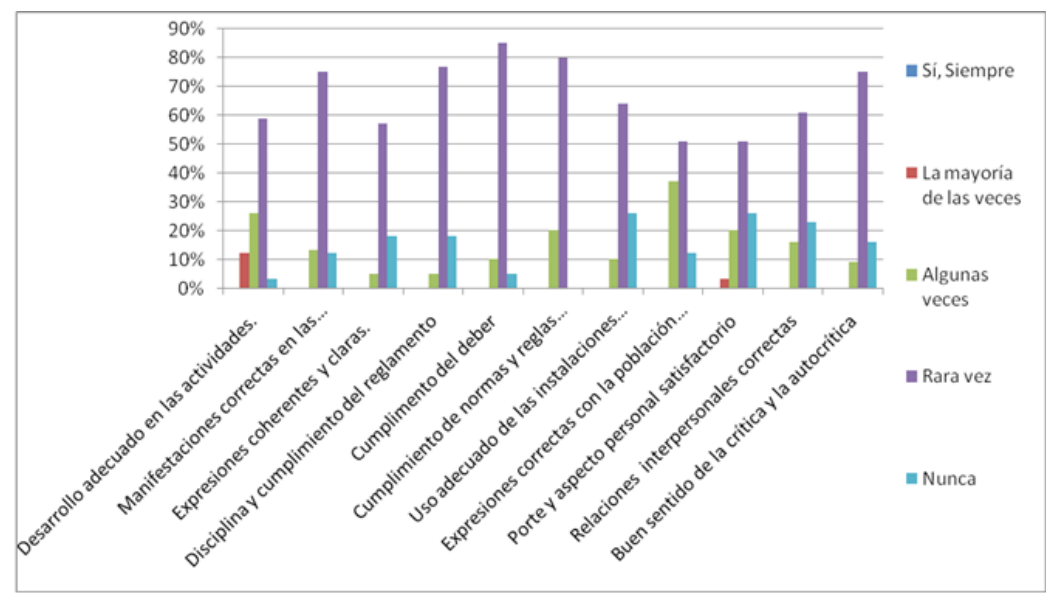


Gráfica 2. Resultados posteriores a la aplicación de la estrategia didáctica

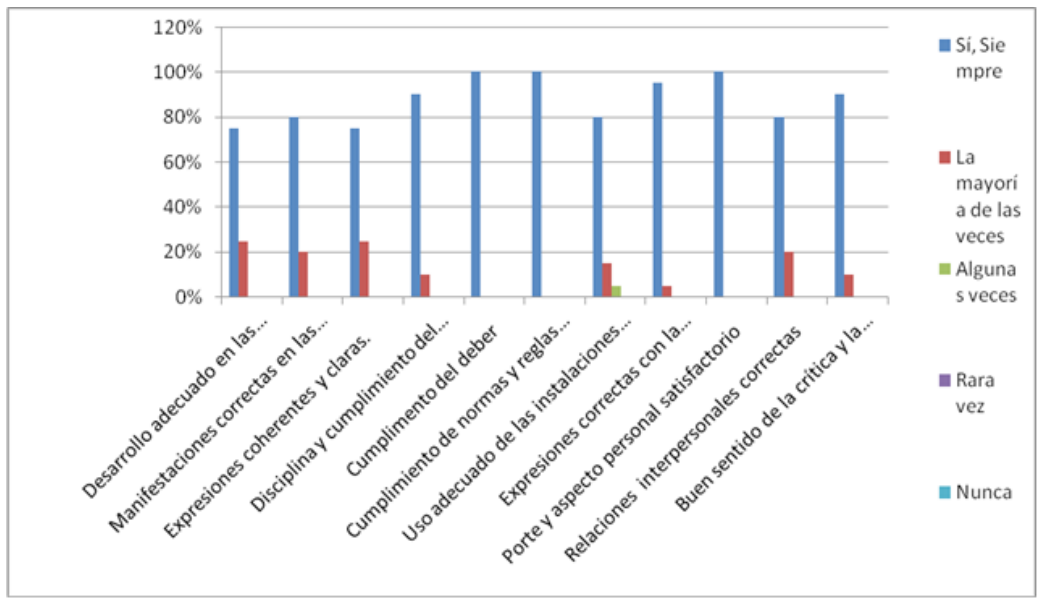

Al comparar estos resultados, se hace evidente que la aplicación de la estrategia didáctica en los estudiantes influyó en el mejoramiento de los modos de actuación en las actividades de la práctica profesional. Esto se constata en la mayoría de los indicadores propuestos para la guía de observación. En conjunto, estos resultados ratifican el valor que tiene la estrategia en la formación del valor responsabilidad en los estudiantes de la citada licenciatura.

\section{Contribución de la estrategia didáctica}

La estrategia didáctica aplicada arrojó los siguientes elementos:

- Una clarificación del valor responsabilidad.

- Incremento del nivel de conocimientos sobre la formación del "valor responsabilidad".

- Concientización teórica sobre el tema de los valores.

-Aumento en el nivel motivacional de los modos de actuación en los diferentes contextos sociales.

-Propuesta de alternativas en el proceso de enseñanza-aprendizaje que contribuyen a mejorar los modos de actuación para la formación del valor responsabilidad mediante la integración de los contenidos.

- Elaboración conjunta de acciones para enfrentar los problemas.

- Consenso en la formación integral del estudiante universitario.

\section{CONCLUSIONES}

El proceso de formación del valor responsabilidad en los estudiantes de la licenciatura en Educación Física y Deporte precisó un análisis de los fundamentos teóricos de la didáctica, así como de las leyes que rigen el comportamiento del proceso, sustentos teóricos que cimentaron la estrategia didáctica. Esta se compone de las etapas de diagnóstico, planeación, ejecución y evaluación, organizadas de manera coherente; no son fijas ni inmutables y pueden ser 
enriquecidas a partir de la reflexión docente y de la práctica pedagógica. Para ello, fue necesario promover el intercambio, las reflexiones y las opiniones partiendo de la valoración de la estrategia.

El diagnóstico nos permitió identificar la necesidad de trabajar con la formación del valor responsabilidad mediante la integración de los contenidos y las actividades de la práctica profesional. El procedimiento metodológico se caracterizó por una estructuración lógica, uniforme y coherente, que se concreta en la formación armónica de los estudiantes de la licenciatura. La elaboración de la estrategia fue resultado de la integración de las posiciones consultadas en la literatura, el criterio de estudiantes y egresados, así como la experiencia de los investigadores; esto, con la cualidad fundamental de mejorar la calidad del proceso de enseñanzaaprendizaje en la profesión.

\section{RECOMENDACIONES}

A partir de los resultados y reflexiones de esta investigación, recomendamos:

- Elaboración de materiales de apoyo para facilitar la implementación de la estrategia didáctica en los diferentes contextos universitarios.

- Socialización de la estrategia didáctica en actividades científico-metodológicas en la comunidad universitaria.

- Propuesta de vías de superación como protagonistas en las transformaciones que genera la integración de contenidos y actividades de la práctica profesional a partir del procedimiento metodológico elaborado en aras de perfeccionar las actividades de las dos academias y la licenciatura.

\section{REFERENCIAS BIBLIOGRÁFICAS}

Addine, F. (2006). Modo de actuación profesional. De la teoría a la práctica. La Habana, Cuba: Academia.

Álvarez de Zayas, C. (1998). Pedagogía como ciencia. La Habana, Cuba: Universidad de La Habana.

Bauzá, E. y Marañón, E. (2012, julio-septiembre). La formación y desarrollo de los valores en la educación superior cubana y su proceso de socialización. Didasc@ lia: Didáctica y Educación, vol. III, núm. 3.

Benítez, A., Díaz, Pozo, Vargas y Núñez (2012). Sistema de tareas para la formación del valor responsabilidad en las clases de idioma inglés. Revista Ciencias Médicas, Cuba, vol. 16, núm. 2, pp. 199-210.

Castro, P., Chacón, N. y Mendoza, L. (2012). La escuela y la formación de valores. Enfoques y experiencias. La Habana: Educación Cubana.

Collado, D. (2005). Transmisión y adquisición de valores a través de un programa de Educación Física basado en el juego motor, en un grupo de alumnos y alumnas de primero de educación secundaria obligatoria. Tesis de doctorado, Universidad de Granada, España.

Cortés, M. y Iglesias, M. (2004). Generalidades sobre metodología de la investigación. Ciudad del Carmen: Universidad Autónoma del Carmen.

Díaz, M. (2011). Una visión de los valores y su formación en el siglo XXI. Revista Avanzada Científica, vol. 14, núm. 2, pp. 1-11.

Domínguez, M. (1996). La formación de valores en la Cuba de los años 90: un enfoque social. La Habana, Cuba: Ediciones Políticas. 
Escámez, J. (2001). Jóvenes, actitudes y responsabilidad. En AA.VV. Familia, juventud y nuestros mayores (pp. 217-233). Galicia: Fundación Caixa Galicia, colección Jornadas, Conferencias, Premios.

Fabelo, J. (2004). Los valores y sus desafíos actuales. Recuperado de www.librosenred.com

Feo, R. (2010). Orientaciones básicas para el diseño de estrategias didácticas. Tendencias Pedagógicas, núm. 16, pp. 220-236.

García, M. (2003). Mediación pedagógica en la educación a distancia. Revista Ciencias Matemáticas, vol. 21, núm. 1.

Gómez, J., Quintana, Medina, Hernández y Beristaín (2013). Educación física y formación de valores en preescolares. Revista Digital de Investigación Educativa Conect@2,pp.1-11.

González, V., Blández, J., López, A. y Sierra, M. (2007). Educación de la responsabilidad en la práctica profesional. La Habana, Cuba: Deportes.

Guevara, B. (2007). ¿Para qué educar en valores? Revista Educación en Valores, vol. 1 , núm. 7.

Izquierdo, C. (2003). Valores de cada día. Caracas, Venezuela: Ediciones San Pablo.

Jiménez Sánchez, L. (2007). La interdisciplinariedad desde un enfoque pedagógico. Tesis doctoral, Editorial Universitaria, La Habana.

León, J. (2011). La formación del valor responsabilidad: un reto histórico. Revista científica digital del centro de investigación y estudios gerenciales (BarquisimetoVenezuela), pp. 192-202.

Licea, D. (2006). La relación interdisciplinaria en el tratamiento de los conocimientos históricos y su aporte al desarrollo de la cultura histórico profesional de los estudiantes de la licenciatura en Educación de Profesores Generales Integrales de Secundaria Básica. Tesis doctoral, Santiago de Cuba.

Moleiro, M. (2001). Relatos para educar en valores. Caracas, Venezuela: Editorial San Pablo.

Navarro, N. (2012). La enseñanza de los valores por el profesorado de educación primaria en el municipio de colima, México. Tesis doctoral, Universidad de Extremadura, España.

Ojalvo, V. (2006). Formación docente para la educación en valores: un reto para la transformación de la Universidad del siglo XXI. La Habana, Cuba: Félix Varela.

Ojalvo, V. (2002). Programa de formación docente para la educación de valores en las universidades. En Programa de Educación en Valores. Madrid, España: OEI. Recuperado a partir de www.oei.es

Paz, A., Comas y Fernández (2011). Consideraciones filosóficas, psicológicas y pedagógicas sobre la formación de valores. Contribuciones a las Ciencias Sociales. Recuperado de www.eumed.net/rev/cccss/11/

Perera, F. (2000). La formación interdisciplinaria de los profesores de ciencias: un ejemplo en el proceso de enseñanza-aprendizaje de la física. Tesis doctoral, La Habana, Cuba.

Reyes, C. (2014). Viktor Frankl: libertad y responsabilidad. México: Trillas.

Sanders, B. (2005). Premisas socioculturales y los valores y creencias en México. En Anuario de Investigación 2004. México: Universidad Autónoma de México.

Sivira, Y. (2011). Programa de actividades físicas y deportivas para contribuir a la educación del valor responsabilidad en escolares en situación de riesgo social de 10-11 años de la escuela bolivariana Genaro Vásquez. Tesis doctoral, Universidad de Ciencias de la Cultura Física y el Deporte "Manuel Fajardo", La Habana, Cuba. 
Torres, M. y Guerrero Soto, A. (coords.) (2012). La investigación educativa en valores en el campo de la corporeidad, el movimiento y la educación física. En Ana Hirsch y Teresa Yurén. La investigación en México en el campo educación y valores. Estado del conocimiento de la década 2002-2011 (pp. 244-287). México: Anuies/ Comie, col. Estados del Conocimiento.

Unesco (2015). Educación física de calidad (EFC). Guía para los responsables políticos. Organización de las Naciones Unidas para la Educación, la Ciencia y la Cultura. Recuperado de www.unesco.org/open-access/terms-use-ccbysa-sp

Valle Lima, D. A. (2007). Metamodelos de la investigación pedagógica. La Habana: Instituto Central de Ciencias Pedagógicas, Ministerio de Educación, Cuba.

Zaldívar, G. (2004). Estudio sobre la educación del valor responsabilidad en los estudiantes universitarios de la cultura física de Holguín. Tesis doctoral, Instituto Superior de Cultura Física "Manuel Fajardo”, Holguín, Cuba. 